\section{Federation University ResearchOnline}

\section{https://researchonline.federation.edu.au}

Copyright Notice

This is the author's preprint version of the following publication:

Abbasi, Kruger, A. Y., \& Théra, M. (2021). Gateaux Differentiability Revisited. Applied Mathematics \& Optimization, 84(3), 3499-3516.

The version displayed here may differ from the final published version.

The final publication is available at Springer via:

https://doi.org/10.1007/s00245-021-09754-y

Copyright ( $)$ The Author(s), under exclusive licence to Springer Science+Business Media, LLC part of Springer Nature 2021 
Noname manuscript No.

(will be inserted by the editor)

\title{
Gateaux differentiability revisited
}

\author{
Malek Abbasi · Alexander Y. Kruger · Michel \\ Théra
}

Received: date / Accepted: date

\begin{abstract}
We revisit some basic concepts and ideas of the classical differential calculus and convex analysis extending them to a broader frame. We reformulate and generalize the notion of Gateaux differentiability and propose new notions of generalized derivative and generalized subdifferential in an arbitrary topological vector space. Meaningful examples preserving the key properties of the original notion of derivative are provided.
\end{abstract}

Keywords Gateaux differentiability · Moreau-Rockafellar subdifferential · Convex function - Directional derivative.

Mathematics Subject Classification (2000) 49J52 - 49J53 · 90C30

\section{Introduction}

Gateaux derivatives are widely used in the calculus of variations, optimization and physics.

According to Laurent Mazliak [7, 8], Gateaux differentiability first appeared in Gateaux's notes $[3,4]$ under the name variation première. Paul Lévy [6] was probably the first to use the name différentielle au sens de Gateaux.

Recall that a mapping $f: X \rightarrow Y$ between Banach spaces is Gateaux differentiable at $\bar{x} \in X$ if, for each $d \in X$, the limit

$$
f^{\prime}(\bar{x} ; d):=\lim _{t \downarrow 0} \frac{f(\bar{x}+t d)-f(\bar{x})}{t}
$$

exists, and $f^{\prime}(\bar{x} ; \cdot)$ is a linear continuous functional on $X$. This functional is called the Gateaux derivative of $f$ at $\bar{x}$. If the limit (1.1) is uniform over $d$ on the unit sphere of $X$, then $f$ is Fréchet differentiable at $\bar{x}$.

Gateaux and Fréchet differentiability coincide for real-valued locally Lipschitz functions on a space in which bounded subsets are relatively compact, in particular, for convex continuous functions in finite dimensions (see, for instance, [12]). Without convexity, the two concepts are different even in finite dimensions.

Malek Abbasi

Department of Mathematics, University of Isfahan, Isfahan, Iran

E-mail: malek.abbasi@sci.ui.ac.ir

Alexander Y. Kruger ORCID 0000-0002-7861-7380

School of Engineering, Information Technology and Physical Science, Federation University Australia, Ballarat,

Vic, Australia

E-mail: a.kruger@federation.edu.au

Michel Théra ORCID 0000-0001-9022-6406

Mathematics and Computer Science Department, University of Limoges

E-mail: michel.thera@unilim.fr 
Gateaux differentiability is easier to check computationally. Being a weaker concept, Gateaux derivative is more universal. For instance, the conventional norm in $\ell^{1}(\mathbb{N})$ is nowhere Fréchet differentiable, but there exists an equivalent norm, which is Gateaux differentiable at all non-zero points (see Phelps [10, Example 1.14(c)]. Note that the conventional norms in $\ell^{1}(\Gamma)$ (with $\Gamma$ uncountable) and $L^{\infty}[0,1]$ are nowhere Gateaux differentiable.

As observed by Moreau [9, Corollary 10.g], if a real-valued convex function is Gateaux differentiable, its Moreau-Rockafellar subdifferential is a singleton. Conversely if a function is finite and continuous at a point and its subdifferential is a singleton, then it is necessarily Gateaux differentiable at this point. The vanishing of the Gateaux derivative is a necessary condition for an extremum.

Throughout the paper, $X$ is a real topological vector space. Its topological dual is denoted by $X^{*}$. Given an extended-real-valued function $f: X \rightarrow \mathbb{R}_{\infty}:=\mathbb{R} \cup\{+\infty\}$, the directional derivative of $f$ at $\bar{x} \in \operatorname{dom} f:=\{x \in X: f(x)<+\infty\}$ in direction $d \in X$ is defined by the (possibly infinite) limit (1.1). We say that $f$ is directionally differentiable at $\bar{x}$ if the limit exists and is finite for all $d \in X$. The mapping $d \mapsto f^{\prime}(\bar{x} ; d)$ is positively homogeneous. If it is linear and continuous, $f$ is Gateaux differentiable at $\bar{x}$. If $f$ is convex, the limit exists and is finite at every point $\bar{x} \in \operatorname{core}(\operatorname{dom} f)$, i.e. when $\cup_{\lambda>0} \lambda(\operatorname{dom} f-\bar{x})=X,{ }^{1}$ and satisfies

$$
f^{\prime}(\bar{x} ; d) \leq f(\bar{x}+d)-f(\bar{x}) .
$$

Furthermore, the function $d \mapsto f^{\prime}(\bar{x} ; d)$ is convex. The subdifferential of $f$ at $\bar{x} \in X$ is the set (cf. [5])

$$
\partial f(\bar{x}):=\left\{x^{*} \in X^{*}:\left\langle x^{*}, d\right\rangle \leq f^{\prime}(\bar{x} ; d) \text { for all } d \in X\right\} .
$$

If $f$ is convex, then the set (1.3) coincides with the Moreau-Rockafellar subdifferential of $f$ at $\bar{x}$ :

$$
\partial f(\bar{x})=\left\{x^{*} \in X^{*}:\left\langle x^{*}, x\right\rangle \leq f(\bar{x}+x)-f(\bar{x}) \quad \forall x \in X\right\} .
$$

In this paper, we revisit some basic concepts and ideas of the classical differential calculus and convex analysis, extending them to a broader frame. Building partially on the tools developed in [1], we reformulate and generalize the notion of Gateaux differentiability and propose new notions of generalized derivative and generalized subdifferential in an arbitrary topological vector space.

The directional derivative (1.1) is replaced by the following limit:

$$
D_{\xi} f(\bar{x})(A):=\lim _{h \downarrow 0} \frac{\xi(f, A(h, \bar{x}))-f(\bar{x})}{h},
$$

where $A:[0,1] \times X \rightrightarrows X$ is a set-valued mapping satisfying $A(0, x)=\{x\}$ for all $x \in X$, and $\xi$ is an extended-real-valued function defined on the product space of the families of all functions $f: X \rightarrow \mathbb{R}_{\infty}$ and all nonempty subsets of $X$, satisfying $\xi(f,\{x\})=f(x)$ for all $f: X \rightarrow \mathbb{R}_{\infty}$ and $x \in X$. Different choices of $A$ and $\xi$ lead to different derivative and subdifferential concepts. Some meaningful examples preserving the key properties of the original notion of derivative are provided in the paper. They include the sup-, average and measure derivatives.

In the next section, we give the main notation, terminology and definitions, as well as some examples. Section 3 is devoted to generalized derivatives and subdifferentials.

\section{Definitions and Examples}

In the rest of the paper, $\mathscr{F}$ and $\mathscr{P}$ stand for the collections of all extended-real-valued functions $f: X \rightarrow \mathbb{R}_{\infty}$ on a real topological vector space $X$ and all nonempty subsets of $X$, respectively. Unless explicitly stated otherwise, linear operations on sets are understood in the Minkowski sense, i.e., if $A, B \subset X$ and $\lambda \in \mathbb{R}$, then $\lambda A:=\{\lambda a: a \in A\}$, and $A+B:=\{a+b$ : $a \in A, b \in B\}$. The constructions defined in this paper employ the following tools:

\footnotetext{
1 If $f$ is convex and lower semicontinuous, core $(\operatorname{dom} f)$ coincides with the interior of dom $f$; see, for instance, [2]
} 
- the family $\mathscr{A}$ of all set-valued mappings $A:[0,1] \times X \rightrightarrows X$ satisfying $A(h, x) \neq \emptyset$ for all $h \in[0,1]$ and $x \in X$, and

$$
A(0, x)=\{x\} \quad \text { for all } \quad x \in X
$$

- the family $\Xi$ of all functions $\xi: \mathscr{F} \times \mathscr{P} \rightarrow \mathbb{R}_{\infty}$ satisfying

$$
\xi(f,\{x\})=f(x) \text { for all } f \in \mathscr{F} \text { and } x \in X .
$$

Observe that $\Xi$ is a convex set.

Convexity of mappings from $\mathscr{A}$ is defined in the usual sense.

Definition 2.1 A mapping $A \in \mathscr{A}$ is convex if

$$
A(\lambda h+(1-\lambda) t, \lambda x+(1-\lambda) y) \subseteq \lambda A(h, x)+(1-\lambda) A(t, y)
$$

for all $\lambda, h, t \in[0,1]$ and $x, y \in X$.

Concavity is defined similarly with the opposite inclusion in the above definition.

Definition 2.2 Let $\mathscr{F}^{\prime} \subseteq \mathscr{F}$ and $\mathscr{P}^{\prime} \subseteq \mathscr{P}$. A function $\xi \in \Xi$ is increasing (respectively, decreasing) on $\mathscr{F}^{\prime} \times \mathscr{P}^{\prime}$ if

$$
\xi(f, U) \leq \xi(f, V) \quad \text { (respectively, } \quad \xi(f, U) \geq \xi(f, V))
$$

for all functions $f \in \mathscr{F}^{\prime}$ and all nonempty subsets $U, V \in \mathscr{P}^{\prime}$ with $U \subseteq V$.

Definition 2.3 Let $A \in \mathscr{A}$ and $\xi \in \Xi$. A function $f \in \mathscr{F}$ is

(i) $\xi$-convex if

$$
\xi(f, \lambda U+(1-\lambda) V) \leq \lambda \xi(f, U)+(1-\lambda) \xi(f, V)
$$

for all nonempty $U, V \subseteq X$ and $\lambda \in[0,1]$;

(ii) $(\xi, A)$-convex if

$$
\xi(f, A(\lambda h+(1-\lambda) t, \lambda x+(1-\lambda) y)) \leq \lambda \xi(f, A(h, x))+(1-\lambda) \xi(f, A(t, y))
$$

for all $x, y \in X$ and $\lambda, h, t \in[0,1]$.

$\xi$-concavity and $(\xi, A)$-concavity are defined similarly with the opposite inequalities in the above definition.

Proposition 2.1 Let $f \in \mathscr{F}, A \in \mathscr{A}$ and $\xi \in \Xi$.

(i) If $f$ is $(\xi, A)$-convex, then it is convex;

(ii) Suppose $A$ is convex and $\xi$ is increasing. If $f$ is $\xi$-convex, then it is $(\xi, A)$-convex.

Proof (i) Suppose $f$ is $(\xi, A)$-convex. Let $x, y \in X$ and $\lambda \in[0,1]$. Then

$$
\begin{aligned}
f(\lambda x+(1-\lambda) y) & =\xi(f,\{\lambda x+(1-\lambda) y\}) \\
& =\xi(f, A(0, \lambda x+(1-\lambda) y)) \\
& \leq \lambda \xi(f, A(0, x))+(1-\lambda) \xi(f, A(0, y)) \\
& =\lambda \xi(f,\{x\})+(1-\lambda) \xi(f,\{y\}) \\
& =\lambda f(x)+(1-\lambda) f(y) .
\end{aligned}
$$

(ii) Suppose $f$ is $\xi$-convex. Let $x, y \in X$ and $\lambda, h, t \in[0,1]$. Then

$$
\begin{aligned}
\xi(f, A(\lambda h+(1-\lambda) t, \lambda x+(1-\lambda) y)) & \leq \xi(f, \lambda A(h, x)+(1-\lambda) A(t, y)) \\
& \leq \lambda \xi(f, A(h, x))+(1-\lambda) \xi(f, A(t, y)) .
\end{aligned}
$$

The proof is completed. 
Example 2.1 Given a function $f \in \mathscr{F}$ and a subset $U \subseteq X$, set

$$
\xi_{\text {sup }}(f, U):=\sup _{u \in U} f(u)
$$

Then

(i) $\xi_{\text {sup }} \in \Xi$;

(ii) $\xi_{\text {sup }}$ is increasing on $\mathscr{F} \times \mathscr{P}$;

(iii) if $f$ is convex, then it is $\xi_{\text {sup }}$-convex.

The first two assertions are obvious. Let $U, V$ be nonempty subsets of $X$ and $\lambda \in[0,1]$. Then

$$
\begin{aligned}
\xi_{\text {sup }}(f, \lambda U+(1-\lambda) V) & =\sup _{u \in U, v \in V} f(\lambda u+(1-\lambda) v) \\
& \leq \sup _{u \in U, v \in V}\{\lambda f(u)+(1-\lambda) f(v)\} \\
& \leq \lambda \sup _{u \in U} f(u)+(1-\lambda) \sup _{v \in V} f(v) \\
& =\lambda \xi_{\text {sup }}(f, U)+(1-\lambda) \xi_{\text {sup }}(f, V) .
\end{aligned}
$$

This proves the third assertion.

Similarly, the function $(f, U) \mapsto \xi_{\text {inf }}(f, U):=\inf _{u \in U} f(u)$ belongs to $\Xi$, and is decreasing. If $f$ is concave, then it is $\xi_{\text {inf }}$-concave.

Let $\sigma: \mathscr{P} \rightarrow X$ denote a selection mapping: $\sigma$ maps each subset $A \in \mathscr{P}$ to some point $a \in A$. In particular, for any $x \in X$, we have $\sigma(\{x\})=x$. The Lebesgue measure on $\mathbb{R}^{n}$ (particularly, on $\mathbb{R}$ ) is denoted by $\lambda^{*}$.

Example 2.2 Given a function $f \in \mathscr{F}$, a subset $A \in \mathscr{P}$ and a selection mapping $\sigma: \mathscr{P} \rightarrow X$, define

$$
\xi_{\sigma}^{*}(f, A):= \begin{cases}\lambda^{*}(f(A))+f(\sigma(A)) & \text { if } f(A) \text { is Lebesque measurable } \\ f(\sigma(A)) & \text { otherwise. }\end{cases}
$$

Since the Lebesgue measure of a singleton is zero, we have $\xi_{\sigma}^{*} \in \Xi$.

Example 2.3 Let $\mathscr{F}^{\prime}$ denote the subfamily of all functions $f \in \mathscr{F}$ such that $f(X)$ is Lebesque measurable. Given a function $f \in \mathscr{F}^{\prime}$, a subset $A \in \mathscr{P}$ and a selection mapping $\sigma: \mathscr{P} \rightarrow X$, define

$$
\xi_{\sigma}^{*}(f, A):=\inf \left\{\lambda^{*}(f(B)): A \subseteq B, f(B) \text { is Lebesque measurable }\right\}+f(\sigma(A)) .
$$

$\xi_{\sigma}^{*}$ is well-defined on $\mathscr{F}^{\prime} \times \mathscr{P}$, and $\xi_{\sigma}^{*} \in \Xi$. If $f(A)$ is Lebesque measurable, then $\xi_{\sigma}^{*}(f, A)=$ $\lambda^{*}(f(A))+f(\sigma(A))$. Furthermore, if $A \subseteq B$ implies that $f(\sigma(A)) \leq f(\sigma(B))$ for all $f \in \mathscr{F}^{\prime}$, then $\xi_{\sigma}^{\star}$ is increasing on $\mathscr{F}^{\prime} \times \mathscr{P}$.

Example 2.4 Given a Lebesque integrable function $f \in \mathscr{F}$, a measurable subset $A \subset \mathbb{R}^{n}$, and a selection mapping $\sigma: \mathscr{P} \rightarrow X$, define

$$
\xi_{i n t}^{\sigma}(f, A):=\int_{A} f d \lambda^{*}+f(\sigma(A))
$$

Then $\xi_{\text {int }}^{\sigma}(f,\{x\})=f(x)$ for all integrable functions $f \in \mathscr{F}$ and all $x \in X$. 
Example 2.5 Let $\mathscr{F}^{\prime} \subseteq \mathscr{F}$ and $\mathscr{P}^{\prime} \subseteq \mathscr{P}$ be the collections of all nonnegative bounded functions $f: X \rightarrow \mathbb{R}$ and all finite or countable subsets of $X$, respectively. Let $\tau_{n}$ denote the set of all permutations on $\{0,1,2, \ldots, n\}$. Given a function $f \in \mathscr{F}^{\prime}$ and an infinite countable set $U:=\left\{x_{0}, x_{1}, x_{2}, \ldots\right\} \in \mathscr{P}^{\prime}$, define

$$
\xi_{\Sigma}(f, U):=\limsup _{n \rightarrow \infty} \max _{\sigma \in \tau_{n}} \sum_{i=0}^{n} 2^{-i n} f\left(x_{\sigma(i)}\right)
$$

For a finite set $U:=\left\{x_{0}, x_{1}, x_{2}, \ldots, x_{n}\right\}$, we consider its infinite countable extension $U^{\prime}:=$ $\left\{x_{0}, x_{1}, x_{2}, \ldots\right\}$ with arbitrary $x_{n+1}, x_{n+2}, \ldots \in X$, and set $\xi_{\Sigma}(f, U):=\xi_{\Sigma}\left(\chi_{U} f, U^{\prime}\right)$, where $\chi_{U}$ denotes the indicator function: $\chi_{U}(x)=1$ if $x \in U$, and $\chi_{U}(x)=0$ if $x \notin U$. Then

(i) $\xi_{\Sigma}(f,\{x\})=f(x)$ for all $f \in \mathscr{F}^{\prime}$ and $x \in X$;

(ii) $\xi_{\Sigma}$ is increasing on $\mathscr{F}^{\prime} \times \mathscr{P}^{\prime}$;

(iii) if $f$ is convex, then it is $\xi_{\Sigma}$-convex.

The first two assertions are obvious. Let $U:=\left\{x_{0}, x_{1}, x_{2}, \ldots\right\}, V:=\left\{y_{0}, y_{1}, y_{2}, \ldots\right\} \subseteq X$ and $\lambda \in[0,1]$. Then $\lambda U+(1-\lambda) V=\left\{u_{i j}:=\lambda x_{i}+(1-\lambda) y_{j}: i, j \in\{0,1,2, \ldots\}\right\}$. Without any loss of generality we can suppose that

$$
f\left(u_{00}\right) \geq f\left(u_{01}\right) \geq \cdots \geq f\left(u_{0 n}\right) \geq f\left(u_{10}\right) \geq \cdots \geq f\left(u_{1 n}\right) \geq \cdots \geq f\left(u_{n 0}\right) \geq \cdots \geq f\left(u_{n n}\right),
$$

for all $n \in \mathbb{N}$. Let $v_{k}:=u_{i j}$ (ordered in the same way as in the above chain of inequalities), where $k:=i+j+n i$. Note that the number of $f\left(u_{i j}\right)$ with $i, j$ varying from zero to $n$ equals $(n+1)^{2}$, and therefore when $i$ and $j$ run over $\{0,1, \ldots n\}$, the index $k$ varies from zero to $(n+1)^{2}-1=n^{2}+2 n$. Thus,

$$
\begin{aligned}
& \xi_{\Sigma}(f, \lambda U+(1-\lambda) V) \\
& =\limsup _{n \rightarrow \infty} \sum_{k=0}^{n^{2}+2 n} 2^{-k\left(n^{2}+2 n\right)} f\left(v_{k}\right) \\
& =\limsup _{n \rightarrow \infty} \sum_{i=0}^{n} \sum_{j=0}^{n} 2^{-(i+j+n i)\left(n^{2}+2 n\right)} f\left(u_{i j}\right) \\
& \leq \limsup _{n \rightarrow \infty} \sum_{i=0}^{n} \sum_{j=0}^{n} 2^{-(i+j+n i)\left(n^{2}+2 n\right)}\left(\lambda f\left(x_{i}\right)+(1-\lambda) f\left(y_{j}\right)\right) \\
& \leq \lambda \limsup _{n \rightarrow \infty} \sum_{i=0}^{n} \sum_{j=0}^{n} 2^{-(i+j+n i)\left(n^{2}+2 n\right)} f\left(x_{i}\right)+ \\
& (1-\lambda) \limsup _{n \rightarrow \infty} \sum_{i=0}^{n} \sum_{j=0}^{n} 2^{-(i+j+n i)\left(n^{2}+2 n\right)} f\left(y_{j}\right) \\
& =\lambda \limsup _{n \rightarrow \infty} \sum_{i=0}^{n} 2^{-i(n+1)\left(n^{2}+2 n\right)} f\left(x_{i}\right) \sum_{j=0}^{n} 2^{-j\left(n^{2}+2 n\right)}+ \\
& (1-\lambda) \limsup _{n \rightarrow \infty} \sum_{j=0}^{n} 2^{-j\left(n^{2}+2 n\right)} f\left(y_{j}\right) \sum_{i=0}^{n} 2^{-i(n+1)\left(n^{2}+2 n\right)} \\
& =\lambda \limsup _{n \rightarrow \infty} \sum_{i=0}^{n} 2^{-i(n+1)\left(n^{2}+2 n\right)} f\left(x_{i}\right) \lim _{n \rightarrow \infty} \frac{1-2^{-(n+1)\left(n^{2}+2 n\right)}}{1-2^{-\left(n^{2}+2 n\right)}}+ \\
& (1-\lambda) \limsup _{n \rightarrow \infty} \sum_{j=0}^{n} 2^{-j\left(n^{2}+2 n\right)} f\left(y_{j}\right) \lim _{n \rightarrow \infty} \frac{1-2^{-(n+1)^{2}\left(n^{2}+2 n\right)}}{1-2^{-(n+1)\left(n^{2}+2 n\right)}} \\
& \leq \lambda \limsup _{n \rightarrow \infty} \sum_{i=0}^{n} 2^{-i n} f\left(x_{i}\right)+(1-\lambda) \limsup _{n \rightarrow \infty} \sum_{j=0}^{n} 2^{-j n} f\left(y_{j}\right)
\end{aligned}
$$




$$
\begin{aligned}
& \leq \lambda \limsup _{n \rightarrow \infty} \max _{\sigma \in \tau_{n}} \sum_{i=0}^{n} 2^{-i n} f\left(x_{\sigma(i)}\right)+(1-\lambda) \limsup _{n \rightarrow \infty} \max _{\sigma \in \tau_{n}} \sum_{j=0}^{n} 2^{-j n} f\left(y_{\sigma(j)}\right) \\
& =\lambda \xi_{\Sigma}(f, U)+(1-\lambda) \xi_{\Sigma}(f, V) .
\end{aligned}
$$

This proves the third assertion.

We now explore some important subfamilies of $\mathscr{A}$.

Example 2.6 Let $\left.\left.\left\{U_{h}: h \in\right] 0,1\right]\right\}$ be a family of neighborhoods of the origin in $X$, and let $U_{0}:=\{0\}$. For all $h \in[0,1]$ and $x \in X$, set $A_{U}(h, x):=\{x\}+U_{h}$. Obviously $A_{U}(0, x)=\{x\}$. Denote by $\mathscr{A}_{U}$ the family of all mappings $A_{U}:[0,1] \times X \rightrightarrows X$ corresponding to the given family $\left\{U_{h}: h \in[0,1]\right\}$. Thus, $\mathscr{A}_{U} \subseteq \mathscr{A}$.

If $X$ is a normed space, and $\mathbb{B}$ its closed unit ball, we take $U_{h}:=h \mathbb{B}$, and write $A_{\mathbb{B}}$ and $\mathscr{A}_{\mathbb{B}}$ instead of $A_{U}$ and $\mathscr{A}_{U}$, respectively. Thus, $A_{\mathbb{B}}(h, x)=\{x\}+h \mathbb{B}$ for all $h \in[0,1]$ and $x \in X$. The mapping $A_{\mathbb{B}} \in \mathscr{A}_{\mathbb{B}}$ is both convex and concave in the sense of Definition 2.1. Indeed, let $x, y \in X$ and $\lambda, h, t \in[0,1]$. Then

$$
\begin{aligned}
A_{\mathbb{B}}(\lambda h+(1-\lambda) t, \lambda x+(1-\lambda) y) & =\{\lambda x+(1-\lambda) y\}+(\lambda h+(1-\lambda) t) \mathbb{B} \\
& =\lambda(\{x\}+h \mathbb{B})+(1-\lambda)(\{y\}+t \mathbb{B}) \\
& =\lambda A_{\mathbb{B}}(h, x)+(1-\lambda) A_{\mathbb{B}}(t, y) .
\end{aligned}
$$

This proves the assertion.

Example 2.7 Let $\Omega$ be a vector space of all continuous functions $\kappa:[0,1] \rightarrow X$ satisfying $\kappa(0)=0$. Given a $\kappa \in \Omega$ and a continuous function $\eta:[0,1] \times X \rightarrow[0,1]$ satisfying

$$
0 \leq \eta(h, x) \leq h \text { for all } h \in[0,1] \text { and } x \in X,
$$

set

$$
A_{\kappa}(h, x):=\{x+\kappa(t): t \in[\eta(h, x), h]\} \quad \text { for all }(h, x) \in[0,1] \times X .
$$

Denote by $\mathscr{A}_{\eta}$ the family of all mappings $A_{\kappa}:[0,1] \times X \rightrightarrows X(\kappa \in \Omega)$, corresponding to the given function $\eta$. Thus, $\mathscr{A}_{\eta} \subseteq \mathscr{A}$.

Proposition 2.2 In Example 2.7, suppose that $(X,\|\cdot\|)$ is a normed space, and $\eta$ satisfies the following condition:

$$
\forall h, \varepsilon>0 \exists x \in X \quad \text { such that } \quad h-\eta(h, x)<\varepsilon .
$$

Set

(i) $A_{\kappa}+A_{\tau}:=A_{\kappa+\tau}$ for all $A_{\kappa}, A_{\tau} \in \mathscr{A}_{\eta}$;

(ii) $\alpha A_{\kappa}:=A_{\alpha \kappa}$ for all $A_{\kappa} \in \mathscr{A}_{\eta}$ and $\alpha \in \mathbb{R}$;

(iii) $\left\|A_{\kappa}\right\|_{\mathscr{A}_{\eta}}:=\sup _{0 \leq t \leq 1}\|\kappa(t)\|$ for all $A_{\kappa} \in \mathscr{A}_{\eta}$.

Then $\mathscr{A}_{\eta}$ is a vector space, and the mapping $A_{\kappa} \mapsto\left\|A_{\kappa}\right\|_{\mathscr{A}_{\eta}}$ defines a norm on $\mathscr{A}_{\eta}$.

Proof Let $\kappa, \tau \in \Omega$ and $A_{\kappa}=A_{\tau}$. To prove that $\mathscr{A}_{\eta}$ is a vector space, it suffices to show that $\kappa=\tau$. Let $\theta \in[0,1]$. Then

$$
\{\kappa(t): t \in[\eta(\theta, x), \theta]\}=\{\tau(t): t \in[\eta(\theta, x), \theta]\} \quad \text { for all } \quad x \in X .
$$

In view of (2.3), there exists a sequence $\left(x_{n}\right) \subseteq X$ such that $\eta\left(\theta, x_{n}\right) \rightarrow \theta$ as $n \rightarrow \infty$. Without loss of generality, we can assume that $\eta\left(\theta, x_{n}\right) \uparrow \theta$. It follows from (2.4) that

$$
\bigcap_{n=1}^{\infty}\left\{\kappa(t): t \in\left[\eta\left(\theta, x_{n}\right), \theta\right]\right\}=\bigcap_{n=1}^{\infty}\left\{\tau(t): t \in\left[\eta\left(\theta, x_{n}\right), \theta\right]\right\} .
$$

Obviously $\left(\left\{\kappa(t): t \in\left[\eta\left(\theta, x_{n}\right), \theta\right]\right\}\right)$ is a decreasing sequence of sets whose diameters converge to zero. The continuity of $\kappa$ implies that $\left\{\kappa(t): t \in\left[\eta\left(\theta, x_{n}\right), \theta\right]\right\}$ is compact for all $n \in$ $\mathbb{N}$. By Cantor's theorem, $\bigcap_{n=1}^{\infty}\left\{\kappa(t): t \in\left[\eta\left(\theta, x_{n}\right), \theta\right]\right\}$ contains exactly one element which, thanks to the continuity of $\kappa$, must equal $\kappa(\theta)$. Similarly $\bigcap_{n=1}^{\infty}\left\{\tau(t): t \in\left[\eta\left(\theta, x_{n}\right), \theta\right]\right\}=$ $\tau(\theta)$. It follows that $\kappa(\theta)=\tau(\theta)$ and, since $\theta \in[0,1]$ is arbitrary, $\kappa=\tau$. Checking that the mapping $A_{\kappa} \mapsto\left\|A_{\kappa}\right\|_{\mathscr{A}_{\eta}}$ defines a norm is trivial. 
Remark 2.1 (i) The linear operations defined in Proposition 2.2 differ from the conventional ones in the Minkowski sense.

(ii) The function $\eta:[0,1] \times X \rightarrow[0,1]$ defined for all $h \in[0,1]$ and $x \in X$ by $\eta(h, x):=\frac{h\|x\|}{\|x\|+1}$ satisfies conditions (2.1) and (2.3).

Example 2.8 (Corresponds to $\Omega$ in Example 2.7 being the family of all functions $[0,1] \ni t \mapsto$ $t u$ determined by vectors $u \in X)$. Given an $\varepsilon \in[0,1]$, the continuous function $\eta_{\varepsilon}:[0,1] \times X \rightarrow$ $[0,1]$, defined by $\eta_{\varepsilon}(h, x):=(1-\varepsilon) h$ for all $h \in[0,1]$ and $x \in X$, satisfies condition (2.1), but not (2.3). Definition (2.2) in this case reduces to

$$
A_{u}^{\varepsilon}(h, x):=\{x+t u: t \in[(1-\varepsilon) h, h]\} \quad \text { for all } \quad(h, x) \in[0,1] \times X .
$$

We denote by $\mathscr{A}_{\varepsilon}:=\left\{A_{u}^{\varepsilon}: u \in X\right\}$. Thus, $\mathscr{A}_{\varepsilon} \subseteq \mathscr{A}$.

It follows from (2.5) that $A_{u}^{0}(h, x)$ is single-valued: $A_{u}^{0}(h, x)=\{x+h u\}$.

Proposition 2.3 Let $\varepsilon \in[0,1]$. In Example 2.8, set

(i) $A_{u}^{\varepsilon}+A_{v}^{\varepsilon}:=A_{u+v}^{\varepsilon}$ for all $A_{u}^{\varepsilon}, A_{u}^{\varepsilon} \in \mathscr{A}_{\varepsilon}$;

(ii) $\alpha A_{u}^{\varepsilon}:=A_{\alpha u}^{\varepsilon}$ for all $A_{u}^{\varepsilon} \in \mathscr{A}_{\varepsilon}$ and $\alpha \in \mathbb{R}$.

Then $\mathscr{A}_{\varepsilon}$ is a vector space. If $(X,\|\cdot\|)$ is a normed space, then the norm on $\mathscr{A}_{\varepsilon}$ can be defined as follows:

(iii) $\left\|A_{u}^{\varepsilon}\right\|_{\mathscr{A}_{\varepsilon}}:=\|u\|$ for all $A_{u}^{\varepsilon} \in \mathscr{A}_{\varepsilon}$.

The linear operations defined above are particular cases of those in Proposition 3.1. However, since $\eta_{\varepsilon}$ does not satisfy condition (2.3), Proposition 3.1 is not applicable even if $X$ is assumed to be a normed space.

Proof Let $u, v \in X$ and $A_{u}^{\varepsilon}=A_{v}^{\varepsilon}$. To prove that $\mathscr{A}_{\varepsilon}$ is a vector space, it suffices to show that $u=v$. It follows from (2.5) with $h=1$ that $\{t u: t \in[1-\varepsilon, 1]\}=\{t v: t \in[1-\varepsilon, 1]\}$. In particular, there exist $t_{1}, t_{2} \in[1-\varepsilon, 1]$ such that $t_{1} u=v$ and $t_{2} v=u$. If $v=0$, then the second equality yields $u=0$. If $v \neq 0$, then the above equalities yield $t_{1} t_{2} v=v$; hence $t_{1}=t_{2}=1$ and $u=v$. Checking that the mapping $A_{u}^{\varepsilon} \mapsto\left\|A_{u}^{\varepsilon}\right\|_{\mathscr{A}_{\varepsilon}}$ defines a norm is trivial.

Remark 2.2 The proof of Proposition 2.3 shows that, for any $\varepsilon \in[0,1]$, the mapping $u \mapsto A_{u}^{\varepsilon}$ defines an isomorphism between $\mathscr{A}_{\varepsilon}$ and $X$.

Proposition 2.4 Let $\varepsilon \in[0,1]$ and $A \in \mathscr{A}_{\varepsilon}$ (as defined in Example 2.8). Then $A$ is convex.

Proof By definition, $A=A_{u}^{\varepsilon}$ for some $u \in X$. Let $x, y \in X, \lambda, h, s \in[0,1]$, and $w \in A_{u}^{\varepsilon}(\lambda h+$ $(1-\lambda) s, \lambda x+(1-\lambda) y)$. By $(2.5), w=\lambda x+(1-\lambda) y+t u$ for some $t \in[(\lambda h+(1-\lambda) s)(1-$ $\varepsilon), \lambda h+(1-\lambda) s]$. Choose numbers $t_{1} \in[h(1-\varepsilon), h]$ and $t_{2} \in[s(1-\varepsilon), s]$ such that $t=$ $\lambda t_{1}+(1-\lambda) t_{2}$. Thus, $w=\lambda\left(x+t_{1} u\right)+(1-\lambda)\left(y+t_{2} u\right) \in \lambda A_{u}^{\varepsilon}(h, x)+(1-\lambda) A_{u}^{\varepsilon}(s, y)$. Вy Definition 2.1, $A$ is convex.

Proposition 2.5 Let $f \in \mathscr{F}, \xi \in \Xi$ and $A \in \mathscr{A}_{0}$ (as defined in Example 2.8 with $\varepsilon=0$ ). Then $f$ is $(\xi, A)$-convex if and only if it is convex.

Proof If $f$ is $(\xi, A)$-convex, then it is convex by Proposition 2.1(i). Suppose that $f$ is convex. Let $x, y \in X$ and $\lambda, h, t \in[0,1]$. Then

$$
\begin{aligned}
\xi(f, A(\lambda h+(1-\lambda) t, & \lambda x+(1-\lambda) y)) \\
& =\xi(f,\{\lambda x+(1-\lambda) y+(\lambda h+(1-\lambda) t) v\}) \\
& =f(\lambda(x+h v)+(1-\lambda)(y+t v)) \\
& \leq \lambda f(x+h v)+(1-\lambda) f(y+t v) \\
& =\lambda \xi(f,\{x+h v\})+(1-\lambda) \xi(f,\{y+t v\}) \\
& =\lambda \xi(f, A(h, x))+(1-\lambda) \xi(f, A(t, y)) .
\end{aligned}
$$

The proof is completed. 


\section{Generalized Derivative and Subdifferential}

In this section, we propose new notions of generalized derivative and generalized subdifferential in an arbitrary topological vector space.

\subsection{Generalized Derivative}

Definition 3.1 Let $f \in \mathscr{F}, \bar{x} \in \operatorname{dom} f, A \in \mathscr{A}$ and $\xi \in \Xi$. If the limit

$$
D_{\xi} f(\bar{x})(A):=\lim _{h \downarrow 0} \frac{\xi(f, A(h, \bar{x}))-f(\bar{x})}{h}
$$

exists (in $\mathbb{R}_{ \pm \infty}$ ), it is called the $\xi$-derivative of $f$ at $\bar{x}$ along $A$, and we say that $f$ is $\xi$-differentiable at $\bar{x}$ along $A$.

If $\mathscr{A}^{\prime} \subseteq \mathscr{A}$ is a linear topological space and the mapping $A \mapsto D_{\xi} f(\bar{x})(A)$ is linear and continuous on $\mathscr{A}^{\prime}$, then we say that $f$ is $\xi$-differentiable at $\bar{x}$ with respect to $\mathscr{A}^{\prime}$.

Example 3.1 Let $(X,\|\cdot\|)$ be a normed space. For all $u, x \in X$ and $h \in[0,1]$, set $A_{u}(h, x):=$ $\left\{x+t u: 0 \leq t \leq h^{2}\right\}$. Observe that $A_{u}(0, x)=\{x\}$; hence, $A_{u} \in \mathscr{A}$. For the function $x \mapsto$ $\|x\|$, using $\xi_{\text {sup }} \in \Xi$ defined in Example 2.1, we have $\xi_{\text {sup }}\left(\|\cdot\|, A_{u}(h, 0)\right)=\sup _{t \in\left[0, h^{2}\right]} t\|u\|=$ $h^{2}\|u\|$. Hence, by Definition 3.1, $D_{\xi_{\text {sup }}}\|\cdot\|(0)\left(A_{u}\right)=0$. Moreover, if $\mathscr{A}^{\prime}:=\left\{A_{u}: u \in X\right\}$ is endowed with a linear structure and norm as in Proposition 2.3, then $\|\cdot\|$ is $\xi_{\text {sup }}$-differentiable at 0 with respect to $\mathscr{A}^{\prime}$ (with the derivative equal 0 ).

Example 3.2 Let $(X,\|\cdot\|)$ be a normed space and, as defined in Example 2.6, $A_{B}(h, x)=$ $\{x\}+h \mathbb{B}$ for all $h \in[0,1]$ and $x \in X$. For a function $f \in \mathscr{F}$ and a point $\bar{x} \in \operatorname{dom} X$, using $\xi_{\text {sup }} \in \Xi$ defined in Example 2.1, we have $\xi_{\text {sup }}\left(f, A_{B}(h, \bar{x})\right)=\sup _{u \in h \mathbb{B}} f(\bar{x}+u)$. Hence, by Definition 3.1, if the limit exists, it has the form

$$
D_{\xi_{\text {sup }}} f(\bar{x})\left(A_{\mathbb{B}}\right)=\lim _{h \downarrow 0} \sup _{u \in h \mathbb{B}} \frac{f(\bar{x}+u)-f(\bar{x})}{h}=\limsup _{h \downarrow 0} \frac{f(\bar{x}+h u)-f(\bar{x})}{h} .
$$

As a consequence, $D_{\xi_{\text {sup }}} f(\bar{x})\left(A_{\mathbb{B}}\right) \geq 0$.

Proposition 3.1 In Example 3.2,

(i) if $f$ is Fréchet differentiable at $\bar{x}$, then $D_{\xi_{\text {sup }}} f(\bar{x})\left(A_{\mathbb{B}}\right)=\|\nabla f(\bar{x})\|$;

(ii) if $f$ is convex, then the limits in (3.2) exist, and

$$
D \xi_{\text {sup }} f(\bar{x})\left(A_{\mathbb{B}}\right) \geq \sup \left\{\left\|x^{*}\right\|: x^{*} \in \partial f(\bar{x})\right\} .
$$

If, additionally, $\operatorname{dim} X<+\infty$ and $\partial f(\bar{x}) \neq \emptyset$, then (3.3) holds as equality.

Proof (i) If $f$ is Fréchet differentiable at $\bar{x}$, then

$$
\lim _{h \downarrow 0} \frac{f(\bar{x}+h u)-f(\bar{x})}{h}=(\nabla f(\bar{x}))^{T} u,
$$

and the limit is uniform in $u$ on the unit sphere of $X$; hence the conclusion.

(ii) Let $f$ be convex. The existence of the limits in (3.2) follows from the monotonicity of the difference quotients involved. If $x^{*} \in \partial f(\bar{x})$, then, by (3.2),

$$
D_{\xi_{\text {sup }}} f(\bar{x})\left(A_{\mathbb{B}}\right) \geq \sup _{u \in \mathbb{B}}\left\langle x^{*}, u\right\rangle=\left\|x^{*}\right\|,
$$

which yields (3.3).

Let $\operatorname{dim} X<+\infty$ and $\partial f(\bar{x}) \neq \emptyset$. If $D_{\xi_{\text {sup }}} f(\bar{x})\left(A_{\mathbb{B}}\right)=0$, the equality holds true trivially. If $\bar{x}$ is a boundary point of $\operatorname{dom} f$, then $\partial f(\bar{x})$ is unbounded (cf. [11, Theorem 23.4]), 
$\sup \left\{\left\|x^{*}\right\|: x^{*} \in \partial f(\bar{x})\right\}=+\infty$, and the equality holds true trivially too. Otherwise, $f$ is Lipschitz continuous near $\bar{x}$ and there exist sequences $h_{k} \downarrow 0$ and $u_{k} \rightarrow 0$ such that $0<\left\|u_{k}\right\| \leq h_{k}, f\left(\bar{x}+u_{k}\right)>f(\bar{x})(k \in \mathbb{N})$ and

$$
\limsup \sup _{h \downarrow 0} \frac{f(\bar{x}+u)-f(\bar{x})}{h}=\lim _{k \rightarrow+\infty} \frac{f\left(\bar{x}+u_{k}\right)-f(\bar{x})}{h_{k}} .
$$

Without loss of generality, $u_{k} /\left\|u_{k}\right\| \rightarrow u \in X$. Set $t_{k}:=\left\|u_{k}\right\|$ and $u_{k}^{\prime}:=t_{k} u(k \in \mathbb{N})$. Then $\left\|u_{k}-u_{k}^{\prime}\right\|=t_{k}\left\|\frac{u_{k}}{\left\|u_{k}\right\|}-u\right\|$, and consequently,

$$
\begin{aligned}
\lim _{k \rightarrow+\infty} \frac{f\left(\bar{x}+u_{k}\right)-f(\bar{x})}{h_{k}} & \leq \lim _{k \rightarrow+\infty} \frac{f\left(\bar{x}+u_{k}\right)-f(\bar{x})}{t_{k}} \\
& =\lim _{k \rightarrow+\infty} \frac{f\left(\bar{x}+t_{k} u\right)-f(\bar{x})}{t_{k}}=f^{\prime}(\bar{x}, u)=\left\langle x^{*}, u\right\rangle
\end{aligned}
$$

for some $x^{*} \in \partial f(\bar{x})$. Hence,

$$
\limsup _{h \downarrow 0} \sup _{u \in h \mathbb{B}} \frac{f(\bar{x}+u)-f(\bar{x})}{h} \leq \sup \left\{\left\|x^{*}\right\|: x^{*} \in \partial f(\bar{x})\right\} .
$$

This proves the claimed equality.

Example 3.3 Let $f \in \mathscr{F}, \bar{x} \in \operatorname{dom} f$ and $A \in \mathscr{A}$. Let $\lambda^{*}$ be the Lebesgue measure on $\mathbb{R}$, $\sigma: \mathscr{P} \rightarrow X$ be a selection mapping and $\xi_{\sigma}^{*} \in \Xi$ be defined as in Example 2.2. Then

$$
\begin{aligned}
D_{\xi_{\sigma}^{*}} f(\bar{x})(A) & =\lim _{h \downarrow 0} \frac{\xi_{\sigma}^{*}(f, A(h, \bar{x}))-f(\bar{x})}{h} \\
& =\lim _{h \downarrow 0} \frac{\lambda^{*}(f(A(h, \bar{x})))+f(\sigma(A(h, \bar{x})))-f(\bar{x})}{h} .
\end{aligned}
$$

If the mapping $A(\cdot, \bar{x})$ satisfies certain continuity property at 0 , then $\sigma(A(h, \bar{x})) \rightarrow \bar{x}$ as $h \downarrow 0$.

Example 3.4 Let $(X, \mathscr{S}, \mu)$ be a measure space. Given a point $\bar{x} \in X$, let

$$
\mathscr{W}_{\bar{x}}:=\{A \in \mathscr{S}: \bar{x} \in A, f(A) \text { is Lebesgue measurable }\} .
$$

Similarly to Example 3.3, we propose the following derivative formula ( $D_{\sigma}^{*}$-derivative):

$$
D_{\sigma}^{*} f(\bar{x}):=\lim _{A \in \mathscr{W}_{\bar{x}}^{\prime}, \mu(A) \downarrow 0} \frac{\lambda^{*}(f(A))+f(\sigma(A))-f(\bar{x})}{\mu(A)}
$$

where $\mathscr{W}_{\bar{x}}^{\prime} \subseteq \mathscr{W}_{\bar{x}}$ and $\sigma: \mathscr{P} \rightarrow X$ is a selection mapping. By letting $\sigma(A)=\bar{x}$ for $A \in \mathscr{W}_{\bar{x}}^{\prime}$, we get

$$
D^{*} f(\bar{x}):=D_{\sigma}^{*} f(\bar{x})=\lim _{A \in \mathscr{W}_{\bar{x}}^{\prime}, \mu(A) \downarrow 0} \frac{\lambda^{*}(f(A))}{\mu(A)} .
$$

For a function $f$ whose range is a null set, one has $D^{*} f(\bar{x})=0$ at every $\bar{x} \in X$. In particular, the $D^{*}$-derivative of the Dirichlet function exists at every $\bar{x} \in \mathbb{R}$ and equals zero.

Example 3.5 In the framework of Example 3.4, we consider the $D^{*}$-derivative of a function $f:[a, b] \rightarrow \mathbb{R}$ at a point $\bar{x} \in] a, b\left[\right.$ with $\mathscr{W}_{\bar{x}}^{\prime}$ denoting the collection of all open intervals in $[a, b]$.

- If $f$ is nondecreasing and continuous, then

$$
D^{*} f(\bar{x})=\lim _{y \downarrow \bar{x}, x \uparrow \bar{x}} \frac{f(y)-f(x)}{y-x} .
$$


- If $f$ is nondecreasing and differentiable and $\mathscr{W}_{\bar{x}}^{\prime}$ denotes the collection of all open intervals centered at $\bar{x}$, then $D^{*} f(\bar{x})=f^{\prime}(\bar{x})$. If $f$ is continuously differentiable, then the equality holds with $\mathscr{W}_{\bar{x}}^{\prime}$ denoting the collection of all open intervals (not necessarily centered at $\bar{x}$ ). Indeed, if $] x, y$ [ is an open interval containing $\bar{x}$, then by the Mean Value Theorem, there exists $\beta(x, y) \in] x, y\left[\right.$ such that $f(y)-f(x)=f^{\prime}(\beta(x, y))(y-x)$. Note that $\beta(x, y)$ approaches $\bar{x}$ as $y \downarrow \bar{x}$ and $x \uparrow \bar{x}$. Thanks to the continuity of $f$ and $f^{\prime}$ it follows that

$$
D^{*} f(\bar{x})=\lim _{y \downarrow \bar{x}, x \uparrow \bar{x}} \frac{f(y)-f(x)}{y-x}=\lim _{y \downarrow \bar{x}, x \uparrow \bar{x}} f^{\prime}(\beta(x, y))=f^{\prime}(\bar{x}) .
$$

Observe that in general $f$ does not have to be continuous at $\bar{x}$. For example, consider the function $f: \mathbb{R} \rightarrow \mathbb{R}$ defined as

$$
f(x):= \begin{cases}x^{2} & x \neq 1 \\ 0 & x=1\end{cases}
$$

The $D^{*}$-derivative of $f$ at $\bar{x}=1$ exists and equals 2 . Indeed, if $] x, y$ [ is an open interval containing 1 , then $f(] x, y[)=] x^{2}, y^{2}\left[\backslash\{1\} \bigcup\{0\}\right.$. Thus, $\lambda^{*}(f(] x, y[))=y^{2}-x^{2}$ and therefore

$$
D^{*} f(1)=\lim _{y \downarrow 1, x \uparrow 1} \frac{y^{2}-x^{2}}{y-x}=2 .
$$

In this example, the discontinuity of $f$ at $\bar{x}$ is a removable one. A function can have a $D^{*}$-derivative at a point at which it has a jump discontinuity. Define $f: \mathbb{R} \rightarrow \mathbb{R}$ as

$$
f(x):= \begin{cases}x & x>1 \\ x-1 & x \leq 1\end{cases}
$$

If $] x, y[$ is an open interval containing 1 , then $f(] x, y[)=] 1, y[\bigcup] x-1,0]$. Thus, $\lambda^{*}(f(] x, y[))=$ $y-1+1-x=y-x$. Therefore,

$$
D^{*} f(1)=\lim _{y \downarrow 1, x \uparrow 1} \frac{y-x}{y-x}=1
$$

Example 3.6 Let $\xi_{i n t}^{\sigma}$ be defined as in Example 2.4. Then

$$
\begin{aligned}
D_{\xi_{\text {int }}^{\sigma}} f(\bar{x})(A) & =\lim _{h \downarrow 0} \frac{\xi_{\text {int }}^{\sigma}(f, A(h, \bar{x}))-f(\bar{x})}{h} \\
& =\lim _{h \downarrow 0} \frac{\int_{A(h, \bar{x})} f d \lambda^{*}+f(\sigma(A(h, \bar{x})))-f(\bar{x})}{h} .
\end{aligned}
$$

We aim to calculate $D \xi_{\text {int }}^{\sigma} f((\bar{x}, \bar{y}))(A)$ for the function $f: \mathbb{R}^{2} \rightarrow \mathbb{R}$ defined as $f(x, y):=x$, where

$$
A(h,(\bar{x}, \bar{y})):=\left\{(u, v): \bar{x}-\frac{\sqrt{h}}{2} \leq u \leq \bar{x}+\frac{\sqrt{h}}{2}, \bar{y}-\frac{\sqrt{h}}{2} \leq v \leq \bar{y}+\frac{\sqrt{h}}{2}\right\} .
$$

Suppose that $\sigma(A(h,(\bar{x}, \bar{y})))=(\bar{x}, \bar{y})$. One can easily check that $\int_{A(h,(\bar{x}, \bar{y}))} f d \lambda^{*}=\bar{x} h$. Thus, $D_{\xi_{\text {int }}} f((\bar{x}, \bar{y}))(A)=\bar{x}=f(\bar{x}, \bar{y})$. If $A(h,(\bar{x}, \bar{y})):=\{(u, v): \bar{x}-h \leq u \leq \bar{x}+h, \bar{y}-h \leq v \leq \bar{y}+h\}$, then one can verify that $D_{\xi_{\text {int }}} f((\bar{x}, \bar{y}))(A)=0$. 
Remark 3.1 Suppose that $\lambda^{*}(A(h, \bar{x})) \neq 0$ for all $h>0$. Similarly to Example 3.6, we propose the following derivative formula:

$$
D_{\text {int }}^{\sigma} f(\bar{x})(A):=\lim _{h \downarrow 0} \frac{\int_{A(h, \bar{x})} f d \lambda^{*}+f(\sigma(A(h, \bar{x})))-f(\bar{x})}{\lambda^{*}(A(h, \bar{x}))} .
$$

For $f: \mathbb{R}^{2} \rightarrow \mathbb{R}$ given by $f(x, y):=x, A(h,(\bar{x}, \bar{y})):=\{(u, v): \bar{x}-h \leq u \leq \bar{x}+h, \bar{y}-h \leq v \leq$ $\bar{y}+h\}$, and $\sigma(A(h,(\bar{x}, \bar{y})))=(\bar{x}, \bar{y})$, one can check that $D_{i n t}^{\sigma} f((\bar{x}, \bar{y}))(A)=\bar{x}=f(\bar{x}, \bar{y})$. Note that the same representation holds if $A(h,(\bar{x}, \bar{y}))$ is a ball centered at $(\bar{x}, \bar{y})$ with radius $h$. These examples make us hope that in general $D_{\text {int }}^{\sigma} f(\bar{x})(A)$ does not depend on $A$, and the derivative (defined by (3.4)) of the integral of a function $f$ equals $f$, mimicking the first part of the Fundamental Theorem of Calculus. This conjecture is currently an open problem.

Example 3.7 Let $X=\mathbb{R}$, and a mapping $A:[0,1] \times \mathbb{R} \rightrightarrows \mathbb{R}$ be defined as follows:

$$
A(h, x):=\left\{x+\frac{(-1)^{k}}{k^{2}}: 0<k^{-1} \leq h\right\} .
$$

Observe that $A(0, x)=\{x\}$; hence $A \in \mathscr{A}$. Next, we consider the function $f:=|\cdot|$ and apply to it the mapping $A$ defined above and the function $\xi_{\Sigma}$ defined in Example 2.5. For all $n \in \mathbb{N}$, we have

$$
\xi_{\Sigma}\left(f, A\left(n^{-1}, 0\right)\right)=\limsup _{m \rightarrow \infty} \sum_{k=0}^{m} 2^{-m k}\left|\frac{(-1)^{n+k}}{(n+k)^{2}}\right| \leq \frac{1}{n^{2}} \lim _{m \rightarrow \infty} \sum_{k=0}^{m} 2^{-m k}=\frac{1}{n^{2}} .
$$

Given a number $h>0$, let $n_{h}$ be the smallest integer such that $n_{h} \geq h^{-1}$. Then

$$
0 \leq \frac{\xi_{\Sigma}(f, A(h, 0))-f(0)}{h} \leq \frac{\xi_{\Sigma}\left(f, A\left(n_{h}^{-1}, 0\right)\right)}{n_{h}^{-1}} \leq n_{h}^{-1} \rightarrow 0
$$

as $h \downarrow$ 0. By Definition 3.1, $D_{\xi_{\Sigma}} f(0)(A)=0$.

Proposition 3.2 Let $A_{d}^{0}$ be defined for all $d \in X$ as in Example 2.8, and let $\xi \in \Xi$. Given a vector $d \in X$, a function $f: X \rightarrow \mathbb{R}_{\infty}$ is $\xi$-differentiable at $\bar{x} \in \operatorname{dom} f$ along $A_{d}^{0}$ if and only if it is differentiable at $\bar{x}$ in the direction $d$. In this case, $D_{\xi} f(\bar{x})\left(A_{d}^{0}\right)=f^{\prime}(\bar{x} ; d)$. Moreover, $f: X \rightarrow \mathbb{R}_{\infty}$ is $\xi$-differentiable at $\bar{x}$ with respect to $\mathscr{A}^{0}:=\left\{A_{d}^{0}: d \in X\right\}$ if and only if it is Gateaux differentiable at $\bar{x}$.

Proof Observe that $\xi\left(f, A_{d}^{0}(h, \bar{x})\right)=\xi(f,\{\bar{x}+h d\})=f(\bar{x}+h d)$. The conclusion follows from Definition 3.1.

Proposition 3.3 Let $A \in \mathscr{A}$ and $\xi \in \Xi$. If $f \in \mathscr{F}$ is $(\xi, A)$-convex then, for any $\bar{x} \in \operatorname{dom} f$, the function

$$
h \mapsto \frac{\xi(f, A(h, \bar{x}))-f(\bar{x})}{h} \quad(h>0)
$$

is nondecreasing, and consequently, $f$ is $\xi$-differentiable at $\bar{x}$ along $A$, and

$$
D_{\xi} f(\bar{x})(A)=\inf _{h>0} \frac{\xi(f, A(h, \bar{x}))-f(\bar{x})}{h} .
$$

Proof Let $0<t \leq h$. Then

$$
\begin{aligned}
\xi(f, A(t, \bar{x})) & =\xi\left(f, A\left(t, \frac{t}{h} \bar{x}+\left(1-\frac{t}{h}\right) \bar{x}\right)\right) \\
& \leq \frac{t}{h} \xi(f, A(h, \bar{x}))+\left(1-\frac{t}{h}\right) \xi(f, A(0, \bar{x}))
\end{aligned}
$$




$$
=\frac{t}{h} \xi(f, A(h, \bar{x}))+\left(1-\frac{t}{h}\right) f(\bar{x}),
$$

and consequently,

$$
\frac{\xi(f, A(t, \bar{x}))-f(\bar{x})}{t} \leq \frac{\xi(f, A(h, \bar{x}))-f(\bar{x})}{h} .
$$

Thus, the function (3.5) is nondecreasing, and therefore the limit (3.1) exists in $\mathbb{R}_{ \pm \infty}$. The remaining assertions follow immediately.

In view of Proposition 2.1(ii), we have the following corollary.

Corollary 3.1 Suppose $A \in \mathscr{A}$ is convex and $\xi \in \Xi$ is increasing. If $f \in \mathscr{F}$ is $\xi$-convex then it is $\xi$-differentiable along $A$ at any $\bar{x} \in \operatorname{dom} f$.

The monotonicity of the function (3.5) established in Proposition 3.3 yields the following result which extends (1.2).

Corollary 3.2 Let $A \in \mathscr{A}$ and $\xi \in \Xi$. If $f \in \mathscr{F}$ is $(\xi, A)$-convex and $\bar{x} \in \operatorname{dom} f$, then

$$
D_{\xi} f(\bar{x})(A) \leq \xi(f, A(1, \bar{x}))-f(\bar{x}) .
$$

\subsection{Generalized Subdifferential}

Let $\mathscr{A}^{\prime} \subseteq \mathscr{A}$ be a linear topological space such that, for any $A \in \mathscr{A}^{\prime}$,

$$
A(h, x)=h A(1, x) \quad \text { for all } h \in] 0,1] \text { and } x \in X,
$$

where $h A$ is determined by the linear structure of the space $\mathscr{A}^{\prime}$ and does not necessarily agree with the conventional scalar multiplication operation in the Minkowski sense. Let a function $\xi \in \Xi$ be given.

The $\xi$-subdifferential of a function $f \in \mathscr{F}$ at $\bar{x} \in \operatorname{dom} f$ with respect to $\mathscr{A}^{\prime}$ is defined by

$$
\partial_{\xi}\left[\mathscr{A}^{\prime}\right] f(\bar{x}):=\left\{A^{*} \in\left(\mathscr{A}^{\prime}\right)^{*}:\left\langle A^{*}, A\right\rangle \leq D_{\xi} f(\bar{x})(A) \quad \text { for all } \quad A \in \mathscr{A}^{\prime}\right\} .
$$

Proposition 3.4 Let $f \in \mathscr{F}$ and $\bar{x} \in \operatorname{dom} f$. If $f$ is $(\xi, A)$-convex for all $A \in \mathscr{A}^{\prime}$, then the following properties are equivalent:

(i) $A^{*} \in \partial_{\xi}\left[\mathscr{A}^{\prime}\right] f(\bar{x})$;

(ii) $\left\langle A^{*}, A\right\rangle \leq \xi(f, A(1, \bar{x}))-f(\bar{x})$ for all $A \in \mathscr{A}^{\prime}$.

Proof The equivalence is a consequence of definition (3.7) in view of Corollary 3.2 and condition (3.6).

Proposition 3.5 Let $f \in \mathscr{F}$ and $\bar{x} \in \operatorname{dom} f$. If $f$ is $(\xi, A)$-convex for all $A \in \mathscr{A}^{\prime}$, then the following assertions hold true:

(i) $\partial_{\xi}\left[\mathscr{A}^{\prime}\right] f(\bar{x})$ is convex and weak ${ }^{*}$ closed.

(ii) If there exist a neighborhood of the origin $\mathscr{N} \subset \mathscr{A}^{\prime}$ and an $\alpha \in \mathbb{R}$ such that $\xi(f, A(1, \bar{x}))<$ $\alpha$ for all $A \in \mathscr{N}$, then $\partial_{\xi}\left[\mathscr{A}^{\prime}\right] f(\bar{x})$ is weak ${ }^{*}$ compact.

(iii) Suppose $\mathscr{F}^{\prime} \subseteq \mathscr{F}$ and $\mathscr{P}^{\prime} \subseteq \mathscr{P}$. If $\bar{x} \in A(1, \bar{x})$ for all $A \in \mathscr{A}^{\prime}$, and $\xi$ is increasing on $\mathscr{F}^{\prime} \times \mathscr{P}^{\prime}$, then $0_{\left(\mathscr{A}^{\prime}\right)^{*}} \in \partial_{\xi}\left[\overline{\mathscr{A}}^{\prime}\right] f(\bar{x})$.

Proof (i) is a consequence of definition (3.7).

(ii) By the hypothesis there exist a neighborhood of the origin $\mathscr{N} \subset \mathscr{A}^{\prime}$ and an $\alpha \in \mathbb{R}$ such that $\xi(f, A(1, \bar{x}))-f(\bar{x})<\alpha$ for all $A \in \mathscr{N}$. Hence, in view of Proposition 3.4,

$$
\partial_{\xi}\left[\mathscr{A}^{\prime}\right] f(\bar{x}) \subseteq\left\{A^{*} \in\left(\mathscr{A}^{\prime}\right)^{*}:\left\langle A^{*}, A\right\rangle \leq \alpha \quad \text { for all } \quad A \in \mathscr{N}\right\} .
$$

The right-hand side of the last inclusion is weak* compact by Alaoglu-Bourbaki theorem. $\partial_{\xi}\left[\mathscr{A}^{\prime}\right] f(\bar{x})$ is weak* closed by (i), and consequently, weak* compact. 
(iii) By the assumption, we have $\xi(f, A(1, \bar{x})) \geq \xi(f,\{\bar{x}\})=f(\bar{x})$. Now apply Proposition 3.4.

We now have a closer look at the space $\mathscr{A}_{\varepsilon} \subset \mathscr{A}(\varepsilon \in[0,1])$ and the function $\xi_{\text {sup }} \in \Xi$ defined in Example 2.8 and Example 2.1, respectively.

Proposition 3.6 Let $f: X \rightarrow \mathbb{R}_{\infty}$ be a convex function, $\bar{x} \in \operatorname{dom} f$, and $\varepsilon \in[0,1]$. Then

$$
\partial_{\xi_{\text {sup }}}\left[\mathscr{A}_{\varepsilon}\right] f(\bar{x})=\left\{x^{*} \in X^{*}:\left\langle x^{*}, u\right\rangle \leq \sup _{1-\varepsilon \leq t \leq 1} f(\bar{x}+t u)-f(\bar{x}) \text { for all } u \in X\right\} .
$$

Proof Recall that, by Proposition 2.3, $\mathscr{A}_{\varepsilon}$ is a linear topological space. Each $A_{u}^{\varepsilon} \in \mathscr{A}_{\varepsilon}$ (see Example 2.8 for the definition) is convex by Proposition 2.4 and satisfies condition (3.6). Indeed, let $u, x \in X$ and $h \in] 0,1]$. Then

$$
\begin{aligned}
A_{u}^{\varepsilon}(h, x) & =\{x+t u: h(1-\varepsilon) \leq t \leq h\} \\
& =\{x+t h u: 1-\varepsilon \leq t \leq 1\}=A_{h u}^{\varepsilon}(1, x)=h A_{u}^{\varepsilon}(1, x) .
\end{aligned}
$$

Since $f$ is convex, it is $\xi_{\text {sup }}$-convex; cf. Example 2.1. Moreover, $\xi_{\text {sup }}$ is increasing. In view of Proposition 2.1(ii), $f$ is $\left(\xi_{\text {sup }}, A_{u}^{\varepsilon}\right)$-convex for all $u \in X$. Given any $u \in X$, we can compute:

$$
\xi_{\text {sup }}\left(f, A_{u}^{\varepsilon}(1, \bar{x})\right)=\xi_{\text {sup }}(f,\{\bar{x}+t u: 1-\varepsilon \leq t \leq 1\})=\sup _{1-\varepsilon \leq t \leq 1} f(\bar{x}+t u)-f(\bar{x}) .
$$

Furthermore, $X$ and $\mathscr{A}_{\varepsilon}$ are isomorphic. Hence, using Proposition 3.4, we arrive at (3.8).

Remark 3.2 (i) When $\varepsilon=0$, the right-hand side of (3.8) reduces to the conventional definition of the Moreau-Rockafellar subdifferential, i.e. $\partial \xi_{\text {sup }}\left[\mathscr{A}_{0}\right] f(\bar{x})=\partial f(\bar{x})$. When $\varepsilon=1$, we obtain from (3.8):

$$
\partial_{\xi_{\text {sup }}}\left[\mathscr{A}_{1}\right] f(\bar{x})=\left\{x^{*} \in X^{*}:\left\langle x^{*}, u\right\rangle \leq \sup _{0 \leq t \leq 1} f(\bar{x}+t x)-f(\bar{x}) \text { for all } u \in X\right\}
$$

i.e. $\partial_{\xi_{\text {sup }}}\left[\mathscr{A}_{1}\right] f(\bar{x})$ coincides with the sup-subdifferential $\partial_{\text {sup }} f(\bar{x})$ defined in [1], while definition (3.1) gives (the limit exists by Proposition 3.3)

$$
D_{\xi_{\text {sup }}} f(\bar{x})\left(A_{d}^{1}\right)=\lim _{h \downarrow 0} \frac{\sup _{0 \leq t \leq h} f(\bar{x}+t d)-f(\bar{x})}{h} \quad(d \in X) .
$$

(ii) Given an $\varepsilon \in[0,1]$ and a $u \in X$, one can consider a symmetric version of (2.5):

$$
A_{u}^{\varepsilon}(h, x):=\{x+t u:|t| \in[(1-\varepsilon) h, h]\} \quad \text { for all } \quad(h, x) \in[0,1] \times X .
$$

In particular, when $\varepsilon=0$, definition (3.1) would give (if $f$ is directionally differentiable at $\bar{x}$ )

$$
D_{\xi_{\text {sup }}} f(\bar{x})\left(A_{d}^{0}\right)=\max \left\{f^{\prime}(\bar{x} ; d), f^{\prime}(\bar{x} ;-d)\right\} \quad(d \in X) .
$$

The last expression coincides with the symmetric derivative $f_{\text {sym }}^{\prime}(\bar{x} ; d)$ defined in [1].

\section{Acknowledgements}

The research was supported by the Australian Research Council, project DP160100854. The authors would like to thank Prof. Jean-Baptiste Hiriart Urruty for attracting their attention to the papers by Laurent Matziak [7,8]. 


\section{References}

1. M. Abbasi, A. K. Kruger, and M. Théra. Enlargements of the Moreau-Rockafellar Subdifferential. https://hal.archives-ouvertes.fr/hal-02484321/file/AKT-Final.pdf, February 2020.

2. J. M. Borwein and Q. J. Zhu. Techniques of variational analysis, volume 20 of CMS Books in Mathematics/Ouvrages de Mathématiques de la SMC. Springer-Verlag, New York, 1922.

3. R. Gateaux. Sur les fonctionnelles continues et les fonctionnelles analytiques. Comptes-Rendus de l?Académie des Sciences de Paris, 157:325-327, 1913.

4. R. Gateaux. Sur la notion d'intégrale dans le domaine fonctionnel et sur la théorie du potentiel. Atti Accad. Naz. Lincei. Rend., 23:310-315, 1914.

5. Alexander D. Ioffe and Vladimir M. Tikhomirov. Theory of Extremal Problems, volume 6 of Studies in Mathematics and Its Applications. North-Holland Publishing Co., Amsterdam, 1979.

6. P. Levy. Analyse Fonctionnelle, volume 5 of Mémoires des Sciences Mathématiques. Gauthier-Villars, Paris, 2015.

7. L. Mazliak. The ghosts of the École Normale. Statist. Sci., 30(3):391-412, 2015.

8. L. Mazliak. René Gateaux, on the century of his death. Mat. Soc. Cult. Riv. Unione Mat. Ital. (I), 8(2):169-190, $429,2015$.

9. J. J. Moreau. Fonctionnelles convexes. Séminaire Jean Leray, (2):1-108, 1966-1967.

10. R. R. Phelps. Convex functions, monotone operators and differentiability, volume 1364 of Lecture Notes in Mathematics. Springer-Verlag, Berlin, second edition, 1993.

11. R. T. Rockafellar. Convex Analysis. Princeton Mathematical Series, No. 28. Princeton University Press, Princeton, N.J., 1970

12. B. Sharp. The differentiability of convex functions on topological linear spaces. Bull. Austral. Math. Soc., 42(2):201-213, 1990 